\title{
Automatic detection method of boundary data of gemstone bearing image based on Fourier transform
}

\author{
LI Cai-hong ${ }^{1}$,ZHU Xiao-Jun ${ }^{2}$ \\ \{lgf147852@tom.com $\left.{ }^{1}, w z 20160401 @ 163 . \mathrm{com}^{2}\right\}$ \\ (1.Changchun Institute of Technology,Changchun 130021,China; \\ 2.NanTong Normal College, Nantong 226010,China)
}

\begin{abstract}
At present, the automatic detection method of boundary data of gem bearing image has the defect of poor detection effect. Therefore, an automatic detection method of boundary data of gem bearing image based on Fourier transform is proposed. Based on infrared imaging technology, the image of gem bearing is obtained, and the image is segmented by Fourier transform algorithm. On this basis, the boundary information of image area is judged by information measurement. According to the result of boundary information measurement of gem bearing image, the boundary data of gem bearing image is extracted by log operator, and the boundary data of image is detected by ant colony algorithm The automatic detection of boundary data of gem bearing image is realized. The experimental results show that compared with the traditional automatic detection method of gem bearing image boundary data, the proposed method greatly improves the detection effect, which fully shows that the proposed automatic detection method of gem bearing image boundary data has better performance.
\end{abstract}

Keywords: Fourier transform;Gemstone bearing;Image;Boundary;Testing;

\section{Introduction}

Gem bearing is a kind of sliding bearing made of gem and other hard materials. A natural or synthetic gem (usually sapphire) can be used as the bearing of the rotating shaft of the meter head or the bearing of other precision instruments by carefully grinding out a conical cavity [1].

Gemstone bearings are mainly used in instruments. The bearing of the instrument bears little load, but it needs high rotation accuracy, good sensitivity and long service life. Gemstone has the characteristics of small friction coefficient, high hardness, corrosion resistance, small thermal expansion coefficient, high compressive strength, and can meet the use requirements of instrument bearing. The materials for making gem shaft include corundum, agate, glass ceramics, etc. corundum is the main body of alumina. They are natural and artificial. Natural corundum has many impurities and uneven texture, so it is widely used in man-made corundum.

Gemstone bearing has certain technical requirements, for example, the conicity of the 
outer circle of the groove gemstone bearing shall not exceed half of the allowable deviation of its outer diameter; the parallelism of the two end faces of the groove gemstone bearing shall not exceed half of the allowable deviation of its height; the perpendicularity of the outer circle of the groove gemstone bearing to the bottom surface shall not be greater than 0.005 ; the coaxiality of the groove gemstone bearing and the outer circle shall not be greater than 0 $02 \mathrm{~mm}$, the spherical bearing is not more than $0.03 \mathrm{~mm}$; the groove bus of the tapered gem bearing is within $1 / 3$ of the groove depth from the bottom; the groove bus of the spherical corundum bearing is within $2 / 3$ of the groove depth from the bottom, etc.

Gem bearing needs high precision, especially the boundary precision of gem bearing. Therefore, higher requirements are put forward for gem bearing detection. Nowadays, infrared imaging technology is mainly used to obtain image boundary data of gemstone bearing, on this basis, to detect whether the gemstone bearing meets the standard. In reference [2], a method of automatic segmentation of SAR image target is proposed. Many algorithms can solve the problem of target segmentation in SAR image, one of them is the grabbing and cutting algorithm. The grab cut algorithm based on graph theory realizes the optimal image segmentation, and transforms the image segmentation problem into the problem of the maximum flow in the computational flow network. However, there are some defects in the automatic detection of boundary data of gem bearing image. In order to solve these problems, an automatic detection method of boundary data of gem bearing image based on Fourier transform is proposed.

Fourier transform is to use a digital computer to calculate in an efficient and fast way, referred to as FFT. Fourier transform was proposed by J.W. KULI and T.W. basis in 1965. By using this algorithm, the number of times of multiplication needed for computing DFT can be greatly reduced. Especially, the more sampling points $n$ are transformed, the more significant the saving of FFT algorithm is. Through the application of Fourier transform algorithm, it can greatly improve the detection effect of gemstone bearing image boundary data automatic detection method, and design simulation contrast experiment to verify the performance of the proposed method.

\section{An automatic detection method for boundary data of gemstone bearing image}

\subsection{Image segmentation of gem bearing}

Based on the infrared imaging technology to obtain the gem bearing image, using the Fourier transform algorithm to segment the image to prepare for the detection of the gem bearing image boundary data, the specific process is as follows [3].

The image segmentation by Fourier transform algorithm is mainly divided into two 
stages: interpolation stage and segmentation stage [4].

Among them, the main operation of the difference stage is to rotate the ray and circle the square. Rotating ray is to obtain ray sampling with even angle in polar coordinate system. It is necessary to rotate ray to get grid point. Its expression is as follows:

$$
B V=\left\{\begin{array}{c}
\xi_{y}=\frac{\pi l}{N} \text { for }-N \leq l<N \\
\xi_{x}=\frac{\pi l}{N} \tan \left(\frac{\pi m}{2 N}\right) \text { for }-\frac{N}{2} \leq l<\frac{N}{2}
\end{array}\right\}
$$

Among them, $B V$ is interpolation grid point; $\xi_{y}$ is Cartesian grid vertical frequency;

$l$ is gem bearing image length; $N$ is gem bearing image matrix order; $\xi_{x}$ is Cartesian grid horizontal frequency; $m$ is algorithm parameter.

The rotating ray diagram is shown in Figure 1.

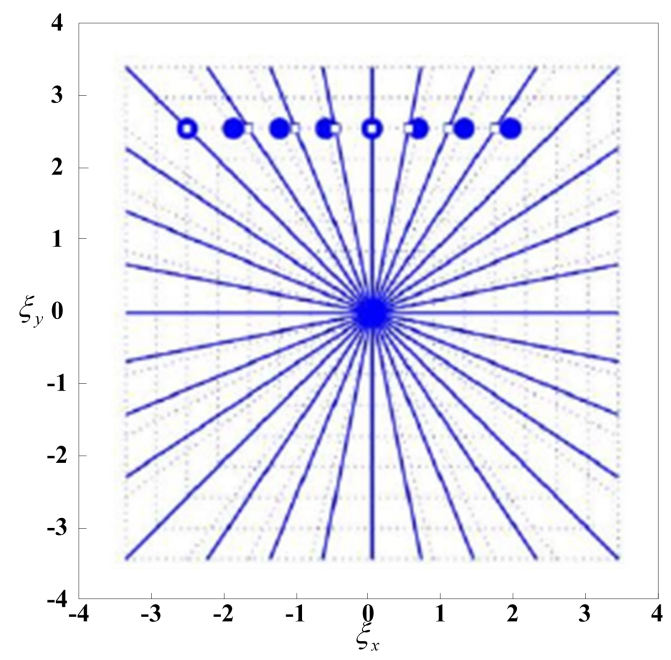

Fig. 1. Schematic diagram of rotating ray

Circle the square to obtain the required concentric circle, which is completed by dividing each ray by a constant along $\xi_{x}$ and $\xi_{y}$, based on its angle. Therefore, set an algorithm parameter $m, R[m]=\sqrt{1+\tan ^{2}\left(\frac{\pi m}{2 N}\right)}$, and the generated grid points are expressed as follows: 


$$
B H=\left\{\begin{array}{c}
\xi_{y}=\frac{\pi l}{N R[m]} \text { for }-N \leq l<N \\
\xi_{x}=\frac{\pi l}{N R[m]} \tan \left(\frac{\pi m}{2 N}\right) \text { for }-\frac{N}{2} \leq l<\frac{N}{2}
\end{array}\right\}
$$

To circle a positive shape is equivalent to an operation along a ray. A group of equidistant points are replaced by a group of new points along the same line in different positions (marked as small orthonormal). This time, the target points are equidistant, but there is a gap between them. The first interpolation stage is applied to trigonometric polynomials to represent the expected accuracy [5].

Circle the square diagram as shown in Figure 2.

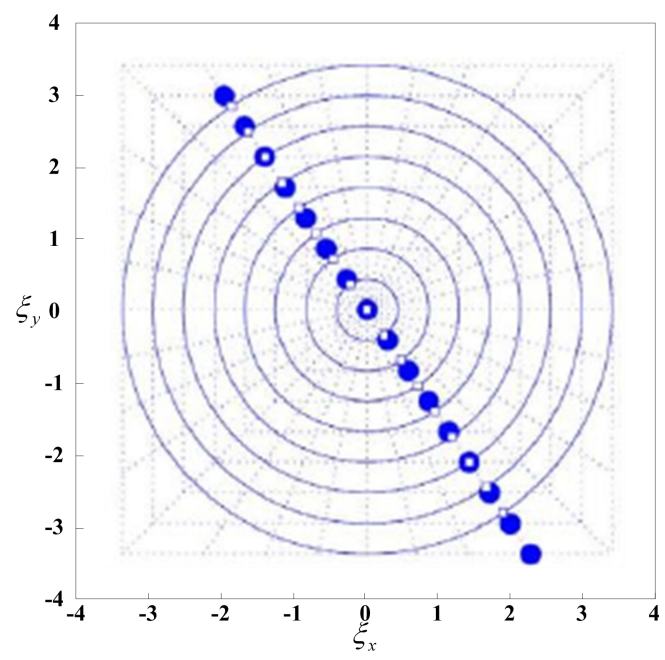

Fig. 2. Circle the square diagram

According to the grid points obtained from the above-mentioned gem bearing image interpolation, the segmentation threshold of gem bearing image is calculated, and the calculation formula is as follows:

$$
T=\frac{B V * B H}{\alpha^{2}}
$$

Among them, a is the segmentation threshold of gem bearing image; $\mathrm{B}$ is the calculation parameter of segmentation threshold.

According to the above formula, the class variance between the target area and the background area of the gem bearing image is obtained, and the expression is as follows:

$$
\delta^{2}(T)=w_{0}\left(\mu_{0}-\mu\right)^{2}+w_{1}\left(\mu_{1}-\mu\right)^{2}
$$


Among them, $w_{0}$ is the proportion of the target area occupying the gem bearing image; $\mu_{0}$ is the average gray level of the target area; $w_{1}$ is the proportion of the background area occupying the gem bearing image; $\mu_{1}$ is the average gray level of the background area.

When $\delta^{2}(T)$ reaches the maximum value, the corresponding threshold $T$ is the best threshold of gem bearing image segmentation. Based on this, the gem bearing image segmentation is realized.

\subsection{Image boundary information measure}

Based on the above segmentation results of gem bearing image, the boundary information of image region can be determined by information measure [6].

In essence, the image noise points and boundary points have obvious characteristics differences: boundary points have structural gray variation and directional gray distribution in their neighborhood; and the gray level of noise points also has variation, but does not have some characteristics of boundary points[7].

The information measure of an image is a measure of the complexity of an image in a small region $\sigma$.generally, $\sigma$ is a circular region with 3-8 pixels. If the image information measure value is large, the image has boundary in area $\sigma$; otherwise, if the image information measure value is small, the image has no boundary in area $\sigma$.

Let the gray level of the image correspond to $f(x, y)$, the coordinate of the current pixel point is $\left(x_{0}, y_{0}\right)$, with point $\left(x_{0}, y_{0}\right)$ as the center, and $\sigma$ is the neighborhood radius, then it meets the following requirements: $\rho$, line $\sigma=\left\{(x, y)|| x-x_{0}|\leq \rho| y-,y_{0} \mid \leq \rho\right\} \quad$ is a straight line passing through the pixel point $L$, and the angle is $\left(0^{\circ}, 180^{\circ}\right)$, and area $\sigma$ is divided into two parts, $\sigma_{1}$ and $\sigma_{2}$, as shown in Figure 3. 


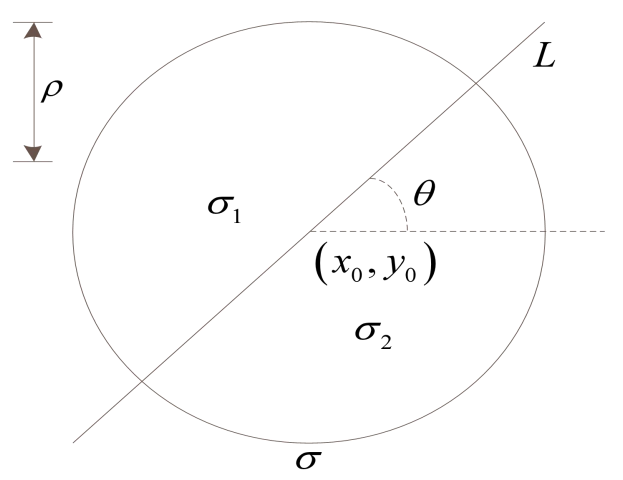

Fig. 3. Area diagram

The image boundary information measure is mainly divided into three aspects: structural boundary information measure, directional boundary information measure and neighborhood consistent boundary information measure.

In gem bearing image, the structural feature of boundary point is a significant difference between boundary point and non boundary point. The structural boundary information measure of point $\left(x_{0}, y_{0}\right)$ is defined as:

$$
C\left(x_{0}, y_{0}\right)=\max \sum_{x, y \in l} g(x, y) /(2 \rho+1)(5)
$$

Where $g(x, y)$ is the gradient amplitude of point $(x, y)$, the calculation formula is:

$$
g(x, y)=\sqrt{\left(\frac{\partial f(x, y)}{\partial x}\right)^{2}+\left(\frac{\partial f(x, y)}{\partial y}\right)^{2}}
$$

The directivity of the boundary point is one of the remarkable characteristics of the boundary point. The expression of the directivity information measure of point $\left(x_{0}, y_{0}\right)$ is:

$$
O\left(x_{0}, y_{0}\right)=\max \left\{\left|f_{\sigma_{1}}-f_{\sigma_{2}}\right|\right\}-\min \left\{\left|f_{\sigma_{1}}-f_{\sigma_{2}}\right|\right\}
$$

The gray distribution of boundary points and non boundary points of an image is different in their respective neighborhoods. The boundary of the image divides the neighborhood of the boundary point into two regions with different gray levels. The gray distribution of non boundary points in their neighborhoods is single. According to this feature, a neighborhood consistent boundary information measure is constructed [8].

Then the neighborhood consistency edge information measure of point $\left(x_{0}, y_{0}\right)$ is: 


$$
R\left(x_{0}, y_{0}\right)=\max \left\{\left|f_{\sigma_{1}}-f_{\sigma_{2}}\right| /[\rho(\rho+1)]\right\}
$$

Through the above analysis, the result of boundary information measure of gem bearing image is $Q=\left\{C\left(x_{0}, y_{0}\right), O\left(x_{0}, y_{0}\right), R\left(x_{0}, y_{0}\right)\right\}$.

\subsection{Image boundary data detection}

Based on the above-mentioned measurement results of boundary information of gem bearing image, LOG operator is used to extract boundary data of gem bearing image.

The schematic diagram of LOG operator is shown in Figure 4.

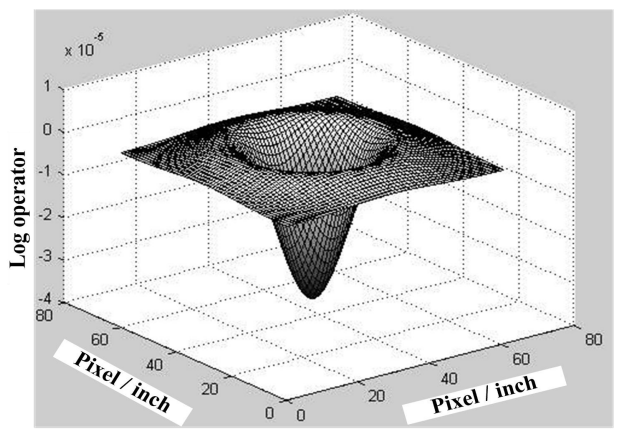

Fig. 4. Schematic diagram of Log Operator

In order to get the output value of LOG operator, Laplacian transform of Gaussian filter function and convolution operation with image are needed. Among them, the two convolution templates that log operators often use are shown in Figure 5.

\begin{tabular}{|c|c|c|c|c|}
\hline-2 & -4 & -4 & -4 & -2 \\
\hline-4 & 0 & 8 & 0 & -4 \\
\hline-4 & 8 & 24 & 8 & -4 \\
\hline-4 & 0 & 8 & 0 & -4 \\
\hline-2 & -4 & -4 & -4 & -2 \\
\hline
\end{tabular}

(a) 


\begin{tabular}{|c|c|c|c|c|}
\hline 0 & 0 & -1 & 0 & 0 \\
\hline 0 & -1 & -2 & -1 & 0 \\
\hline-1 & -2 & 16 & -2 & -1 \\
\hline 0 & -1 & -2 & -1 & 0 \\
\hline 0 & 0 & -1 & 0 & 0 \\
\hline
\end{tabular}

(b)

Fig. 5. Schematic diagram of convolution template of Log Operator

\subsection{Image Boundary Data Detection}

According to the above extraction results, the image boundary data is detected based on ant colony algorithm, and the specific detection process is as follows.

Using ant colony algorithm to detect image boundary data, the first problem to be solved is how to transform the problem of image boundary data detection into a mathematical model which can be detected by ant colony algorithm. In the process of image boundary data detection, the gray gradient of image pixels is mostly selected as the heuristic information of ants. The basic idea of transformation is to use ant colony algorithm to transform image boundary data detection problem into combinatorial optimization problem. Images are considered maps with many pixels. Each pixel is the node that ants choose. From a certain node, ants can move in the neighborhood of pixels. According to the pheromone intensity and heuristic guide function of neighborhood pixels, ants select the pheromone concentration and heuristic guide function, take the point with the maximum calculated transfer probability as the node to be selected in the next crawling, release the pheromone on the previous node, and update the pheromone matrix with the pheromone formula, so the pheromone concentration on the boundary data is significantly higher than other nodes Point, so that most ants can quickly find the boundary data of the image [9].

The steps of detecting image boundary data based on ant colony algorithm are as follows:

Step 1: set the initial value of basic information.

In the detection image of $M \times N, m$ ants are randomly distributed. The size of $m$ is the square root of the number of pixels in the image, i.e. $m=\sqrt{M \times N}$. Set the total number of iterations $Z$ and $q_{\max }$ of ant cycle, and the initial value of pheromone $\tau^{0}=0.0001$. In order to ensure the efficiency of the algorithm, if $\tau_{i j} \geq T$, then $V(i, j)$ 
is the image boundary data, otherwise it is not.

Step 2: ant B selects the rules of the next node.

In the algorithm, each node moved by ant $k$ is considered as an iterative process. Therefore, the total number of times of ant $k$ 's movement $q$ is the total number of iterations of ant $k$ 's movement. In the iterative process of ant $k$, the transfer probability function from the current node $(i, j)$ to the next node $(n, m)$ is:

$$
P_{(i, j)(n, m)}^{k}=\left\{\begin{array}{cc}
\frac{\left[\tau_{i j}^{(n-1)}\right]^{\beta}\left[\eta_{i j}\right]^{\gamma}}{\sum_{(i, j)}\left[\tau_{i j}^{(n-1)}\right]^{\beta}\left[\eta_{i j}\right]^{\gamma}} & (i, j) \in \operatorname{allow}_{(i, j)(9)}^{k} \\
0 & \text { Other }
\end{array}\right.
$$

Among them, $\tau_{i j}{ }^{(n-1)}$ is the pheromone value of the ant at the end of the $n-1$ iteration at node $(i, j) ; \beta$ is the pheromone influence factor, whose size has a certain impact on the probability of ant path selection; $\eta_{i j}$ is the heuristic guidance function of the ant at node $(i, j)$, which is determined by the size of the gray value of the 8 neighborhood of the node; $\gamma$ is the influence factor of the heuristic function, which is determined by the size of the gray value of the node The size of ants has an effect on the probability of selecting neighborhood points with high gradient value.

Step 3: update rule of pheromone.

The pheromone value of each node in the gem bearing image needs to be updated locally and globally.

When ant $k$ completes a move, it needs to update the pheromone locally, that is, after ant $k$ moves to the next node $(i, j)$, it needs to update the pheromone value of node $(i, j)$. The calculation formula is as formula (10); otherwise, it will not be updated. 


$$
\left\{\begin{array}{c}
\tau_{i j}{ }^{n}=(1-\rho) \cdot \tau_{i j}{ }^{\left({ }^{n-1)}\right.}+\rho \cdot \Delta \tau_{i j}{ }^{k} \\
\Delta \tau_{i j}{ }^{k}=\frac{V_{e}\left(I_{(i, j)}\right)}{C}
\end{array}\right.
$$

Among them, $\tau_{i j}{ }^{n}$ is the concentration of pheromone on node $(i, j)$ after ants perform $n$ searches; $\rho$ is the volatility coefficient of pheromone, with a value of 0.076 ; $\Delta \tau_{i j}{ }^{k}$ is the increment of node $(i, j)$ pheromone of the $k$ ant after the current cycle.

When all ants complete a cycle, the pheromone values of all pixels in the image shall be calculated according to formula (11).

$$
\tau_{i j}{ }^{n}=(1-\psi) \cdot \tau_{i j}{ }^{(n-1)}+\psi \cdot \Delta \tau_{i j}{ }^{k}
$$

Among them, $\psi$ is the decay coefficient of the whole pheromone matrix, which is 0.05 .

The advantage of using global update: ant colony algorithm is an algorithm that uses probability method to calculate. In order to make ants better find the edge of the image, avoid falling into the local optimum, concentrate too much on the edge with too much pheromone content, and lose some scattered and small edge information, thus expanding the scope of ant search [10].

Through the above process, the expression of boundary data detection of gem bearing image is obtained as follows:

$$
X_{i}=\frac{\sum T * Q}{P_{(i, j)(n, m)}^{k} \times \tau_{i j}{ }^{n}}
$$

In conclusion, the automatic detection of boundary data of gem bearing image is realized, which provides a new technical support for gem bearing detection.

\section{Test results}

The above-mentioned process realizes the design of automatic detection method of boundary data of gem bearing image based on Fourier transform, but whether it can solve the problems existing in the existing methods is still uncertain, so the simulation contrast experiment is designed. The specific experimental process is as follows.

\subsection{Construction of Experimental Environment}

In order to ensure the smooth progress of the experiment, the experimental environment is set up, as shown in Figure 6. 


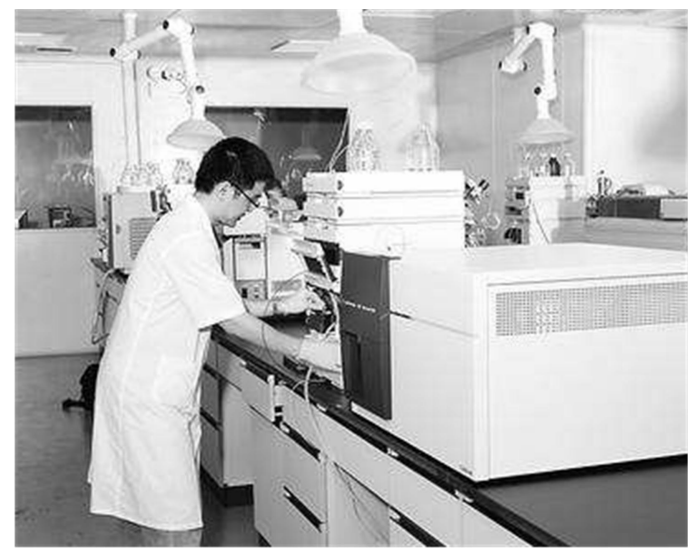

Fig. 6. Schematic diagram of experimental environment

\subsection{Selection of experimental indicators}

The position accuracy, width and continuity of image boundary can be used to judge the performance of image boundary data detection. In this paper, a standard measure $F$ is introduced to evaluate the performance of boundary data detection, which is defined as:

$$
F=\frac{1}{\max \left(I_{D}, I_{L}\right)} \sum_{i=1}^{I_{D}} \frac{1}{1+\zeta I_{i}} \text { (13) }
$$

Among them, $I_{D}$ is the number of ideal boundary pixels; $I_{L}$ is the number of actual detected boundary pixels; $\zeta$ is the penalty factor between the value $[0,1] ; I_{i}$ is the distance between the detected boundary point and the nearest ideal boundary point.

In the experiment, the test image of the measurement standard is used, as shown in Figure 7. The boundary information of the test image is known. Different methods are used to detect the test image. Compared with the boundary characteristics of the standard test image, the boundary detection performance index $F$ of each algorithm can be obtained. The value of $F$ can reflect the performance of boundary detection. The closer $F$ value is to 1 , the better the effect of boundary data detection is. 


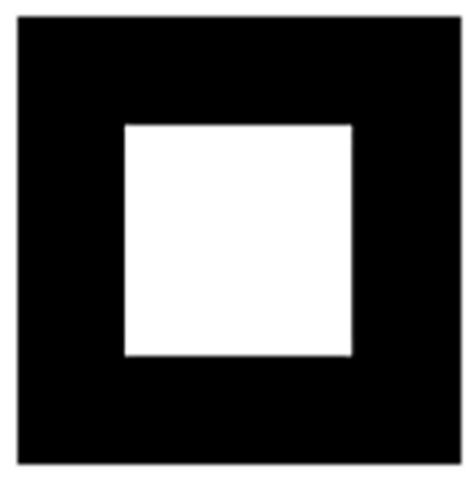

Fig. 7. Standard chart of boundary data detection performance test

\subsection{Analysis of experimental results}

The comparison of measurement $F$ is shown in Table 1.

Table 1 Measurement $F$ comparison table

\begin{tabular}{ccc}
\hline $\begin{array}{c}\text { Number of } \\
\text { experiments }\end{array}$ & Literature [2] method & Propose method \\
\hline 10 & -2.13 & 0.89 \\
20 & 2.13 & 0.78 \\
30 & 0.00 & 0.9 \\
40 & 0.12 & 0.99 \\
50 & 2.46 & 1.00 \\
60 & 2.56 & 1.01 \\
70 & 2.33 & 1.01 \\
80 & 3.00 & 1.00 \\
90 & 3.02 & 1.03 \\
100 & 3.06 & 1.06 \\
\hline
\end{tabular}

As shown in Table 1, the measurement $F$ of the proposed method is closer to 1 , indicating that the detection effect of the proposed method is better.

The experimental results show that: compared with the traditional automatic detection method of gem bearing image boundary data, the proposed automatic detection method of gem bearing image boundary data greatly improves the detection effect, which fully shows that the proposed automatic detection method of gem bearing image boundary data has better performance.

\section{Concluding remarks}

Because the traditional automatic detection method of boundary data of gem bearing 
image is not effective, this paper proposes an automatic detection method of boundary data of gem bearing image. This method greatly improves the detection effect and provides a new technical support for the detection of gem bearing. However, there is still room for improvement in the detection effect of the proposed method, which needs to be further optimized.

\section{References}

[1] Chen Yong, Zhan Di, Liu huanlin, et al.: Enhancement Algorithm for Low-lighting Images Based on Physical Model and Boundary Constraint. Journal of Electronics \& Information Technology, Vol. 39, no. 12, pp. 2962-2969 (2017)

[2] Chen Hua, Guo Wei, Yan Jingwen.: Synthetic aperture radar image target segmentation method based on boundary and texture information. Journal of Image and Graphics, Vol. 24, no. 6, pp. 882-889 (2019)

[3] Zhang Yingting, Liu Bingzhong, Chen wenfen, et al.: Comparison of positioning accuracy between personalized polyurethane foam with wing boards and negative pressure vacuum bag in radiotherapy for lung cancer. Chinese Journal of Radiation Oncology, Vol. 27, no. 3, pp. 299-302 (2018)

[4] Meng Wenjun, Zhang Sicong, Dan Ziyan, et al.: Dynamic Assessment of Rolling Bearing Reliability by State Visualization Based on Class Statistics. Journal of Xi'an Jiaotong University, Vol. 52, no. 6, pp. 23-29 (2018)

[5] Yuan qiongwen, Yu Lei, Liu Yan.: Research on LCI Image Lesion Detection About Fully Convolutional Network Algorithm. Journal of Signal Processing, Vol. 35, no. 3, pp. 451-459 (2019)

[6] Chen Siwen, Liu Yujiang, Liu Dong, et al.: AlexNet Model and Adaptive Contrast Enhancement Based Ultrasound Imaging Classification. Computer Science, Vol. 46, no. 06, pp. 146-152 (2019)

[7] Tenghua, Ren Jianli, Ran Haitao, et al.: The application value of three-dimensional echocardiographic automated quantification technique for assessing left heart chamber volumes and function. Chinese Journal of Ultrasonography, Vol. 28, no. 3, pp. 192-199 (2019)

[8] Deng Rui, Xu Zhuofei, Xu Qianqian, et al.: Fault Diagnosis Method of Printing Press Bearings Based on Image Analysis of Sound Field. Packaging Engineering, Vol. 39, no. 9, pp. 151-157 (2018)

[9] Ma Xiang, Chu Yingying, Chen Yunjie.: Medical Image Segmentation Based on Active Contour Model of Spatial Information Entropy. Control Engineering of China, Vol. 25, no. 11, pp. 2010-2016 (2018)

[10] Yang Jun, Zhao Lin.: The text extraction algorithm based on multi-feature detection and support vector regression. Optical Technique, Vol. 44, no. 5, pp. 609-616 (2018) 